\title{
HPLC Method for Chemical Fingerprinting of Guggul (Commiphora wightii) - Quantification of E- and Z- Guggulsterones and Detection of Possible Adulterants
}

Authors

Affiliations
Rida Ahmed ${ }^{1,3,5}$, Yan-Hong Wang ${ }^{1}$, Zulfiqar Ali ${ }^{1}$, Troy J. Smillie ${ }^{1}$, Ikhlas A. Khan ${ }^{1,2,4}$

The affiliations are listed at the end of the article

\author{
Key words \\ - Commiphora wightii \\ - Burseraceae \\ - guggul \\ - HPLC \\ - quantitative analysis
}

\section{Abstract \\ $\nabla$}

A reversed-phase high-performance liquid chromatography method was developed for the chemical fingerprinting of Commiphora wightii gum resin (guggul). This method was also used for the quantification of E- and Z-guggulsterones in different $C$. wightii gum resin samples. The analysis was conducted on a high-performance liquid chromatography system with a reversed-phase column using a gradient elution comprised of

\section{Introduction}

$\nabla$

Commiphora wightii (Arn.) Bhandari (Burseraceae), syn. Commiphora mukul (Hook.) Engl., is an important medicinal plant which grows in the arid regions of Pakistan and India. Due to its significant pharmacological properties, the gum resin of $C$. wightii (guggul) has been extensively used in Asian indigenous medicines. Scientific studies have proven the significance of guggul as a hypolipidemic and hypoglycemic agent [1,2]. Several dietary supplements claiming to contain guggul extracts are commercially available in dosage forms of tablets and capsules. The demand of guggul has been increasing with the increasing trend of its use in botanicals in Europe, Japan, and North America.

C. wightii has been declared as a red-listed endangered plant species in Rajasthan state (India) because of its poor growth and unsustainable harvesting practices [1]. Currently, the world demand for guggul is largely met from Pakistan, with a reported serious source depletion. The supply of authentic guggul cannot meet the demand owing to its poor growth. This leads to the possibility of adulteration, either deliberately or accidentally. The probability of deliberate adulteration is more since there is gap in demand and supply. The availability of gum resins from different species of water and acetonitrile with $0.1 \%$ acetic acid, and a run time of $50 \mathrm{~min}$ at $38^{\circ} \mathrm{C}$. The calibration curve of E- and Z-guggulsterones showed good linearity with a standard correlation coefficient $>0.999$, which is within the established range $(0.5-250 \mu \mathrm{g} / \mathrm{mL})$. Twelve marker compounds were selected and successfully analyzed by this method in 22 C. wightii gum resin samples along with 9 gum resin samples of possible adulterant species. monly available gum resin in both Pakistan and India. This led us to develop the chemical fingerprinting method of guggul by using HPLC to evaluate the authenticity of its raw material and finished products.

Guggul contains different classes of compounds including triterpenes, steroids, and lignans. Several scientific studies have shown that the pharmacological properties associated with guggul are due to the E- and Z-guggulsterones, while other compounds may synergistically enhance its overall activity [4-6]. Previously developed HPLC and UPLC methods for guggul have only used Eand Z-guggulsterones as standard marker compounds for the qualitative and quantitative analysis of raw guggul and its finished products [7-9]. 
Interestingly, E- and Z-guggulsterones are not specific to $C$. wightii. These compounds have been found from the gum resins of Ailanthus grandis Prain (Simaroubaceae). Z-guggulsterone has also been identified from Ailanthus malabarica DC. as a major compound $[10,11]$. Therefore, the authentication of raw material and/or finished pharmaceutical preparations of $C$. wightii gum resin needs the chemical profile of other secondary metabolites which are specific to $C$. wightii along with E- and Z-guggulsterones. In order to unambiguously identify the source of guggul, a new chemical fingerprinting method was developed by using different classes of standard compounds, including triterpenes, steroids, and lignans. This method assesses the quality of guggul based on both qualitative and quantitative aspects and can differentiate between gum resins of $C$. wightii and its closely related plant species that are commonly available in Pakistan and India. This chemical fingerprinting method is unique, as so far no such chemical fingerprinting approach has been reported for guggul.

\section{Results and Discussion}

$\nabla$

During the method development, an extraction protocol was validated before the optimization of chromatographic conditions because sample preparation is a crucial step in the development of analytical procedures. The extraction method of gum resin samples of $C$. wightii was evaluated for different solvents, temperatures, and extraction times. Methanol, acetonitrile, and acetone have been tested for the extraction of $C$. wightii gum resin (NCNPR \#4998). The liquid chromatography with ultraviolet detection (LC-UV) analysis of different samples prepared using methanol, acetone, and acetonitrile showed similar results for methanol and acetonitrile, whereas acetone was found inefficient. Finally, methanol was selected because it is cost effective compared to acetonitrile. For the optimization of temperature, samples were extracted using methanol at ambient temperature and $55^{\circ} \mathrm{C}$ during sonication, respectively. LC-UV results revealed no significant difference for the main components at two different temperatures. Therefore, extraction was carried out at the ambient temperature $\left(\sim 27^{\circ} \mathrm{C}\right)$. Extraction time was tested for $15 \mathrm{~min}, 20 \mathrm{~min}$, and $30 \mathrm{~min}$, respectively, under sonication, but LC-UV results showed no major difference for different sonication times. Twenty minutes were finally used as the sonication time. The gum resin samples were extracted four times under the optimized extraction protocol using methanol at the ambient temperature, and each extraction time was set for $20 \mathrm{~min}$. LC-UV analysis was carried out at $243 \mathrm{~nm}$ and $205 \mathrm{~nm}$, owing to the detection of compounds 1-7 and 8-12, respectively.

In the developed method, 12 standard compounds were selected for chemical fingerprinting analysis of $C$. wightii gum resin ( Fig. 1). These standard compounds were isolated from a commercial $C$. wightii gum resin during the phytochemical investigation of guggul in our previous study [3]. The mixture of 12 standard compounds (standard mixture-12) was prepared after mixing $50 \mu \mathrm{L}$ of $1.0 \mathrm{mg} / \mathrm{mL}$ of each standard compound. Different solvent system, $\mathrm{C}-18$ columns, and column temperatures have been optimized for choosing the best chromatographic conditions. Acetonitrile/water and acetonitrile/methanol/water were used in different ratios along with various reversed-phase C18 columns, including Phenomenex Synergi Hydro-RP (150 mm × $4.6 \mathrm{~mm}$ i.d.; $4 \mu \mathrm{m})$, Phenomenex Synergi Fusion-RP $(150 \mathrm{~mm} \times 4.6 \mathrm{~mm}$ i.d.; $4 \mu \mathrm{m})$, and Phenomenex Synergi Polar-RP $(150 \mathrm{~mm} \times 4.6 \mathrm{~mm}$ i.d.; $4 \mu \mathrm{m})$, under numerous temperatures ranging from $35^{\circ} \mathrm{C}$ to $45^{\circ} \mathrm{C}$.

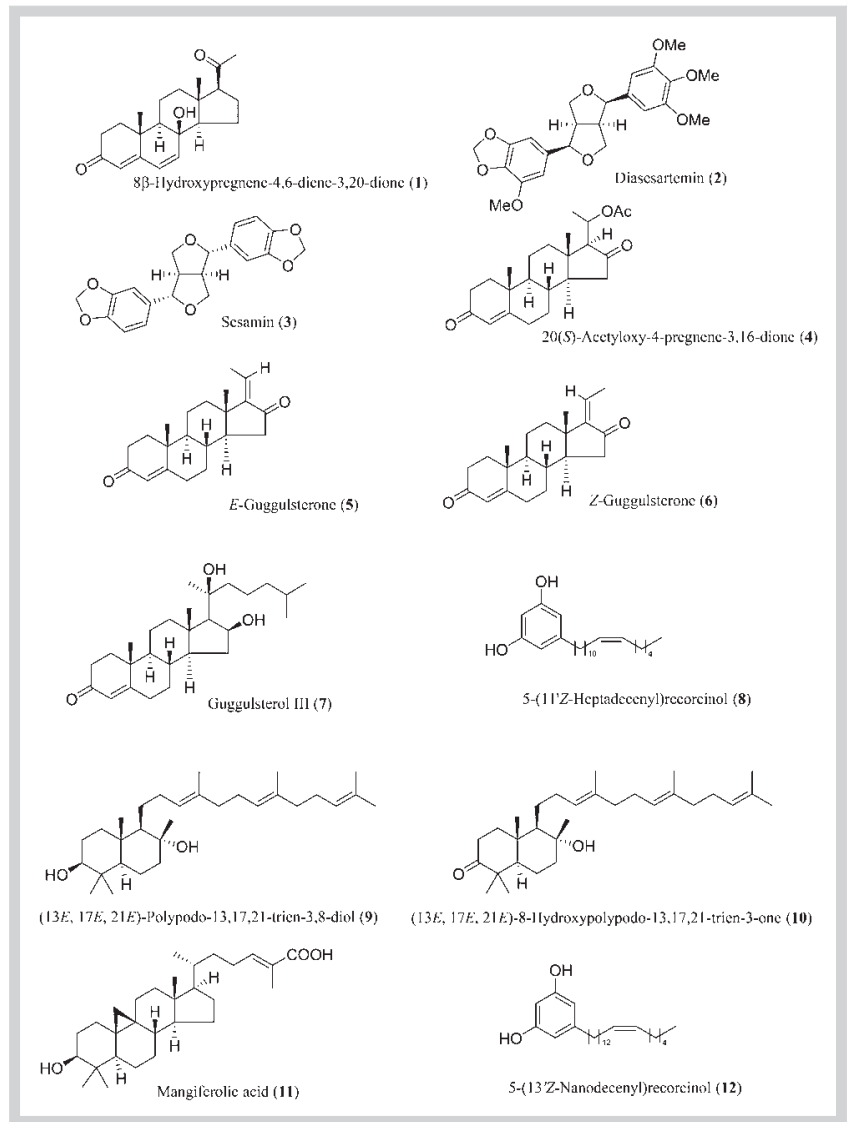

Fig. 1 Structures of standard compounds 1-12.

The best results were obtained with the Phenomenex Synergi Hydro- $R P(150 \mathrm{~mm} \times 4.6 \mathrm{~mm}$ i. d.; $4 \mu \mathrm{m})$ column at $38^{\circ} \mathrm{C}$. The mobile phase was composed of water with $0.1 \%$ acetic acid (A) and acetonitrile containing $0.1 \%$ acetic acid (B) with a gradient elution from $47 \%$ B to $100 \%$ B in $46 \mathrm{~min}$. Acetic acid was used to facilitate the peak separation (see details in Material and Methods). Standard mixture-12 and the gum resin sample (NCNPR \#4998) were analyzed during the optimization of chromatographic conditions. Twenty-two samples of $C$. wightii gum resin were analyzed by using a newly developed chemical fingerprinting method. LC-UV data showed some similarities and differences among all the guggul samples. Fig. $\mathbf{2}$ a, $\mathbf{b}$ represents the chemical profiles of five samples of guggul including three authentic (NCNPR \#4997, \#2567, \#7764) and two commercial samples (NCNPR \#4998, \#5782) both from Pakistan and India. LC-UV profiles of NCNPR \#5782 from Pakistan and NCNPR \#7764 from India showed the presence of nine standard compounds, whereas NCNPR \#4997, \#4998, and \#2576 revealed the presence of eight standard compounds. The analysis of all twenty-two guggul samples revealed the presence of four marker compounds including E-guggulsterone (5) and Z-guggulsterone (6) along with the two major triterpenes (13E,17E,21E)-ploypodo-13,17,21-trien-3,8-diol (9) and (13E,17E,21E)-8-hydroxypolypodo-13,17,21-trien-3-one (10), whereas compounds $\mathbf{8}, \mathbf{1 1}$, and $\mathbf{1 2}$ were absent in twenty-one guggul samples and found only in one commercial guggul sample, which was used in our previous study [3]. The absence of compounds $\mathbf{8}, \mathbf{1 1}$, and 12 in all of the samples except one guggul sample indicated that these compounds do not belong to the gum resin of $C$. wightii and can be the result of possible adulteration. 

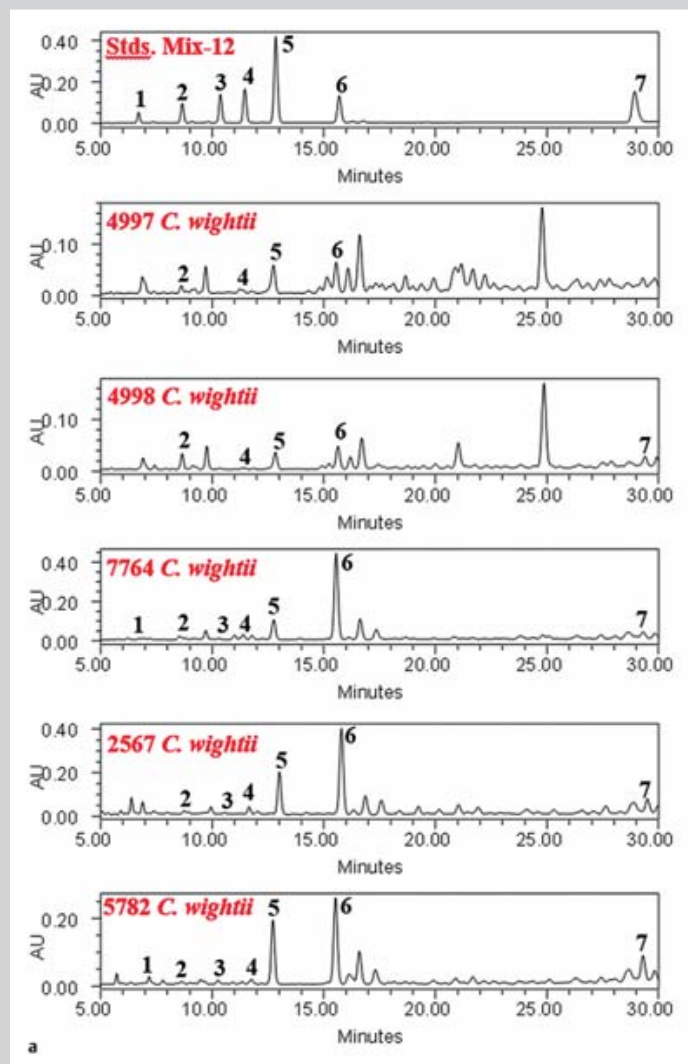
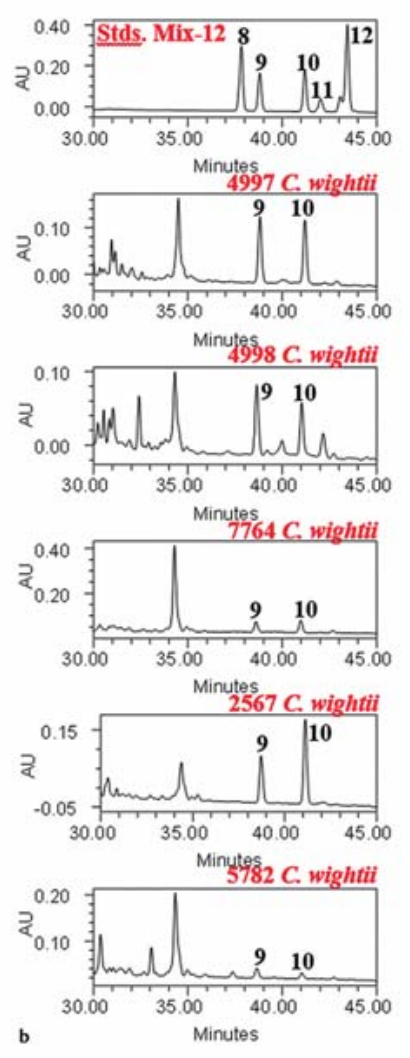

Fig. 2 a HPLC-UV chromatograms of the standards and extracts of $C$. wightii at $243 \mathrm{~nm}$ (RTs between 5 and 30 min). b HPLC-UV chromatograms of the standards and extracts of $C$. wightii at $205 \mathrm{~nm}$ (RTs between 30 and $45 \mathrm{~min}$ ). (Color figure available online only.)
To identify the possible adulterants, gum resins of Acacia nilotica (L.) Willd. ex Del. (Fabaceae), Melia azadirachta L. (Meliaceae), M. indica, and Commiphora myrrha (Nees) Engl. were obtained from different sources in Pakistan, India, and the USA. These gum resins are commonly available in both Pakistan and India. Nine samples were analyzed and compared with standard mixture-12 for the detection of any potential adulteration in the commercial guggul sample.

- Fig. 3 a, b represents the LC-UV data of commercially available C. wightii gum resin sample from Pakistan (EtOAc extract, which was used in our previous phytochemical study, [3]) and four possible adulterant gum resin samples (NCNPR \#2092, \#7761, \#7763, and \#5773). The LC-UV data of commercially available C. wightii gum resin sample from Pakistan (EtOAc extract) showed the presence of all the 12 standard compounds (1-12). LC-UV profiles of the gum resins of A. nilotica, M. azadirachta, and C. myrrha did not show the presence of any of the standard compounds. Only the LC-UV profile of NCNPR \#7761, which is an authentic sample of $M$. indica, showed the presence of standard compounds $\mathbf{8}$, $\mathbf{1 1}$, and 12, which confirms the adulteration of $M$. indica in one of the commercially available $C$. wightii gum resin samples (EtOAC extract). Selected parts of the LC-UV chromatograms are presented (retention times between 5-30 min at $243 \mathrm{~nm}$ and 30-45 min at $205 \mathrm{~nm}$ ) in 0 Figs. 2 and 3.

This method was also validated for the standard parameters such as specificity, accuracy, precision, linearity, limit of detection (LOD), and limit of quantification (LOQ). All standard compounds kept in a $-20^{\circ} \mathrm{C}$ refrigerator were found to be stable for up to 30 days, and no changes were observed with UV spectra, peak areas, and the appearance of any extra peaks. The specificity of the HPLC method was determined by injecting the individual standard compounds, wherein no interference was observed for any of the components. The precision of the developed procedure was assessed by carrying out three independent assays on three days. Three C. wightii gum resin samples (NCNPR \#4998) were extracted under the extraction protocol on three different days and injected in duplicate. High reproducibility was obtained in results after multiple injections with a low standard error. The RSD of assay results obtained in interday and intraday studies is listed in Table 1 . The RSD was less than $2 \%$ with a maximum $1.49 \%$ in the interday assays for E- and Z-guggulsterones, which indicated the good precision of the developed method.

Known quantities of each E- and Z-guggulsterone, i.e., $500 \mu \mathrm{g}$, were spiked in the $C$. wightii gum (NCNPR \#5781) resin sample to measure the accuracy of the assay method. $500 \mu \mathrm{L}$ of both $\mathrm{E}$ - and Z-guggulsterones $(1 \mathrm{mg} / \mathrm{mL}$ ) were spiked in two different samples of $C$. wightii gum resin, weighing $102.3 \mathrm{mg}$ and $102.0 \mathrm{mg}$, respectively. Spiked samples were dried under vacuum and extracted by using the standard extraction protocol. The extracted samples were injected and the data was analyzed to find out the percentage of recovery of the two ketosteroids. The percentage of recovery of E- and Z-guggulsterones was found to be $96.5 \%$ and $100.3 \%$, respectively.

Linear calibration plots for the related substances were obtained at ten different concentration levels (between $0.5-250 \mu \mathrm{g} / \mathrm{mL}$ ) in duplicate. Linear regression analysis of the calibration plot of Eand Z-guggulsterones yielded equations $\mathrm{Y}=(4.55 \mathrm{e}+004) \mathrm{X}+$ $(2.30 \mathrm{e}+004)$ and $\mathrm{Y}=(4.13 \mathrm{e}+004) \mathrm{X}+(1.46 \mathrm{e}+004)$, respectively, and the calibration data indicated the linearity $\left(r^{2}>0.999\right)$ of the detector response for both guggulsterones by the LC-UV method. LOD and LOQ were determined by injecting a series of dilute solutions with known concentrations. LOD and LOQ were defined as the signal-to-noise ratio equal to 3 and 10, respectively. The 

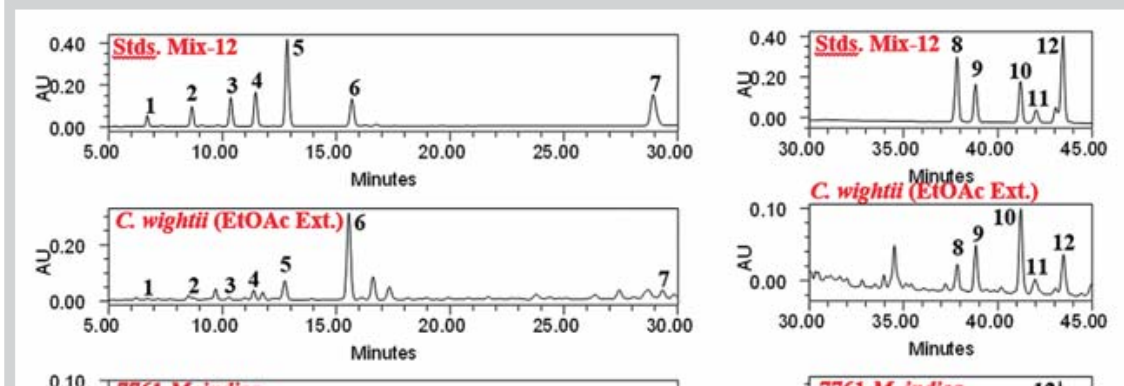

Fig. 3 a HPLC-UV chromatograms of the standards, EtOAc extract of $C$. wightii, and possible adulterants at $243 \mathrm{~nm}$ (RTs between 5 and $30 \mathrm{~min}$ ). b HPLC-UV chromatograms of the standards, EtOAC extract of $C$. wightii, and possible adulterants at $205 \mathrm{~nm}$ (RTs between 35 and $45 \mathrm{~min}$ ).

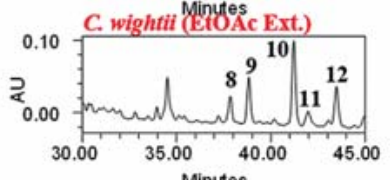
(Color figure available online only.)
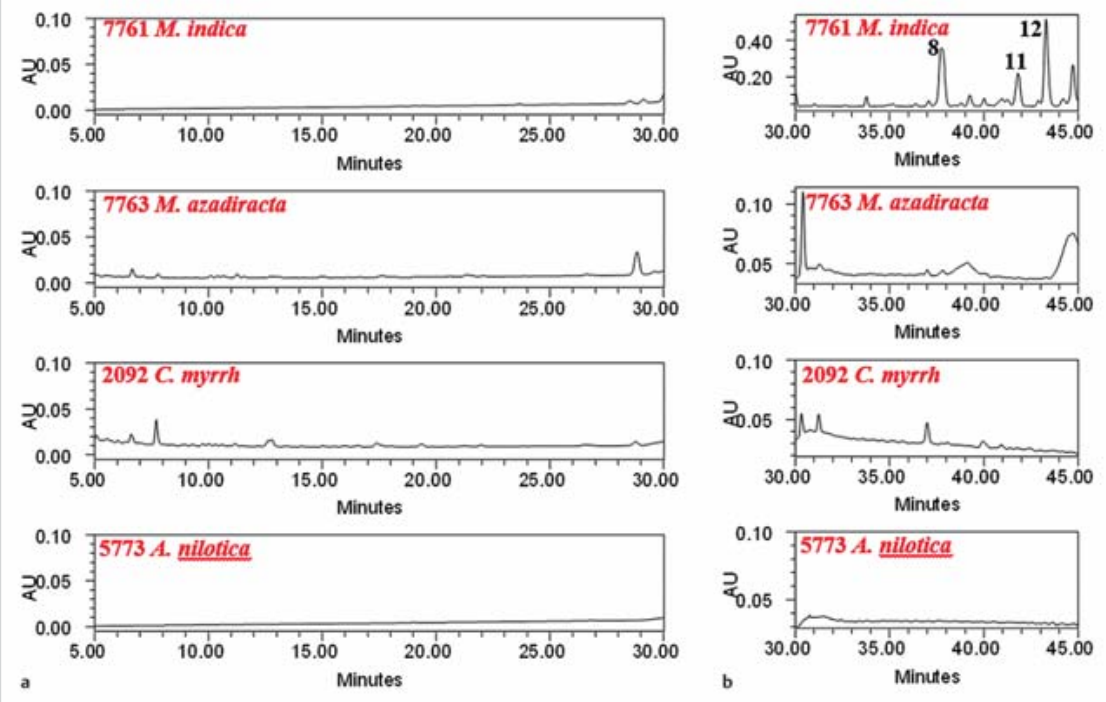

LOD and LOQ for E- and Z-guggulsterones were found to be $0.1 \mu \mathrm{g} / \mathrm{mL}$ and $0.5 \mu \mathrm{g} / \mathrm{mL}$, respectively.

The chemical fingerprinting method developed in this study has been validated for the quantification of E- and Z-guggulsterones in all of the $C$. wightii gum resin samples. The percentage content of E- and Z-guggulsterones of all of the twenty-two samples of guggul is shown in 0 Table 2. The amount of E- and Z-guggulsterones ranges from $0.051 \%$ to $0.867 \%$ and $0.063 \%$ to $4.623 \%$, respectively. There was a considerable variation in the amount of both guggulsterones in all of the $C$. wightii gum resin samples. The overall amount of Z-guggulsterone was found to be more compared to E-guggulsterone in most of the $C$. wightii gum resin samples. This can be because of several factors, such as habitat, soil conditions, weather, time of collection, and age of the plant at the time of harvesting.

The newly developed HPLC chemical fingerprinting method was applied for both qualitative and quantitative analysis of the gum resin of $C$. wightii. Twelve standard compounds have been successfully identified by using this method in all samples, which include twenty-two $C$. wightii gum resin samples and nine samples of possible adulterant gum resins. The quantitative analysis of Eand Z-guggulsterones showed variations in the amount of guggulsterones in several samples. Chemical fingerprinting studies showed that out of the twelve standard compounds, nine compounds were specific to $C$. wightii gum resin, whereas three compounds, 8,11 , and 12 , belonged to $M$. indica. This confirmed the adulteration of $M$. indica gum resin in one of the commercial C. wightii gum resin samples. The results of the analysis revealed that the newly developed HPLC chemical fingerprinting method can be effectively used for the quality control and authentication of herbal raw materials and/or finished pharmaceutical preparations of guggul.
Table 1 Intraday and interday precision data of E- and Z-guggulsterone content (\%, mg/100 mg sample) in NCNPR \#4998.

\begin{tabular}{|c|c|c|c|c|}
\hline \multirow[t]{2}{*}{ Analyte } & \multicolumn{3}{|c|}{ Intraday $(n=3)$} & \multirow[t]{2}{*}{ Interday $(\mathbf{n}=9)$} \\
\hline & Day 1 & Day 2 & Day 3 & \\
\hline E-guggulsterone & $\begin{array}{l}0.907 \\
(0.21)\end{array}$ & $\begin{array}{l}0.928 \\
(0.78)\end{array}$ & $\begin{array}{l}0.911 \\
(1.49)\end{array}$ & $0.916(1.35)$ \\
\hline Z-guggulsterone & $\begin{array}{l}1.176 \\
(0.23)\end{array}$ & $\begin{array}{l}1.206 \\
(0.75)\end{array}$ & $\begin{array}{l}1.195 \\
(1.22)\end{array}$ & $1.192(1.30)$ \\
\hline
\end{tabular}

\% Relative standard deviation given in parenthesis

\section{Materials and Methods}

\section{Instrumentation and chromatographic conditions}

Samples were analyzed on a Waters Alliance 2695 HPLC system (Waters Corp.), 6000 A pumps with quaternary solvent manager, U6 K injector, 680 automated gradient controller, and 2996 photodiode array detector connected to a computerized data station using Waters Empower 2 software. A Synergi-hydro column $(150 \mathrm{~mm} \times 4.6 \mathrm{~mm} ; 4 \mu \mathrm{m})$ from Phenomenex (Torrance) was used as the stationary phase. The column temperature was maintained at $38^{\circ} \mathrm{C}$. An LC-18 $(2 \mathrm{~cm})$ guard column (Phenomenex, Torrance) was used prior to the Synergi-hydro column. A binary mobile phase system comprised of water (A) and acetonitrile (B), both with $0.1 \%$ acetic acid, were used for the study. A flow rate of $1.0 \mathrm{~mL} / \mathrm{min}$ was maintained during the analysis by using the following gradient elution: $53 \% \mathrm{~A} / 47 \% \mathrm{~B}$ to $28 \% \mathrm{~A} / 72 \% \mathrm{~B}$ in 27 min, continually increase B to $81 \%$ B in 1 min, then to $93 \%$ B and $100 \% \mathrm{~B}$ in next $17 \mathrm{~min}$ and $1 \mathrm{~min}$, respectively. After each run, washing for $5 \mathrm{~min}$ with $100 \%$ acetonitrile was carried out along with an equilibration period of $15 \mathrm{~min}$. The injection vol- 
Table 2 E- and Z-guggulsterones \% of content in different C. wightii gum resins and related genera samples.

\begin{tabular}{|c|c|c|c|}
\hline \multirow[t]{2}{*}{ Sample code } & \multirow{2}{*}{$\begin{array}{l}\text { Weight of } \\
\text { the sample } \\
\text { (mg) }\end{array}$} & \multicolumn{2}{|c|}{$\begin{array}{l}\text { \% Content } \\
\text { (mg/weight of the product) }\end{array}$} \\
\hline & & $\begin{array}{l}\text { E-guggul- } \\
\text { sterone }\end{array}$ & $\begin{array}{l}\text { Z-guggul- } \\
\text { sterone }\end{array}$ \\
\hline 4997 & 102.3 & 0.144 & 0.128 \\
\hline 4998 & 102.2 & 0.091 & 0.211 \\
\hline 4999 & 100.2 & 0.134 & 0.292 \\
\hline 2567 & 100.5 & 0.455 & 1.162 \\
\hline 5772 & 101.7 & 0.051 & 0.074 \\
\hline 5774 & 100.9 & 0.558 & 2.352 \\
\hline 5775 & 101.7 & 0.091 & 2.090 \\
\hline 5776 & 100.9 & 0.198 & 1.471 \\
\hline 5777 & 104.8 & 0.143 & 0.168 \\
\hline 5778 & 104.8 & 0.224 & 0.592 \\
\hline 5779 & 104.8 & 0.244 & 1.962 \\
\hline 5780 & 104.7 & 0.378 & 0.568 \\
\hline 5781 & 100.2 & 0.362 & 0.903 \\
\hline 5782 & 102.7 & 0.446 & 0.710 \\
\hline 5783 & 101.3 & 0.236 & 0.331 \\
\hline 5784 & 100.9 & 0.797 & 2.411 \\
\hline 5785 & 101 & 0.074 & 0.063 \\
\hline 5786 & 101.1 & 0.288 & 0.907 \\
\hline 3639 & 104.4 & 0.455 & 3.173 \\
\hline 4985 & 104 & 0.238 & 0.409 \\
\hline 7764 & 103.5 & 0.241 & 1.219 \\
\hline $\begin{array}{l}\text { C. wightii } \\
\text { EtOAc extract }\end{array}$ & $1 \mathrm{mg} / \mathrm{mL}$ & 0.867 & 4.623 \\
\hline 2092 C. myrrha & 104.4 & ND & ND \\
\hline 3550 C. myrrha & 102.2 & ND & ND \\
\hline $5000 \mathrm{M}$. indica & 103.3 & ND & ND \\
\hline 7761 M. indica & 102.5 & ND & ND \\
\hline 7762 M. indica & 104 & ND & ND \\
\hline 6517 M. indica & 104.7 & ND & ND \\
\hline 7763 M. azadiracta & 104.7 & ND & ND \\
\hline 7760 A. nilotica & 101.1 & ND & ND \\
\hline 5773 A. nilotica & 101.2 & ND & ND \\
\hline
\end{tabular}

ND: not detected

ume was $10 \mu \mathrm{L}$. Peaks were detected at UV wavelengths of 205 and $243 \mathrm{~nm}$ and were assigned by spiking the samples with standard compounds, comparison of UV spectra, and from the retention times.

\section{Chemicals}

Twelve standard compounds, $8 \beta$-hydroxypregnene-4,6-diene3,20-dione (1), diasesartemin (2), sesamin (3), 20(S)-acetyloxy4-pregnene-3,16-dione (4), E-guggulsterone (5), Z-guggulsterone (6), guggulsterol III (7), 5-(11'Z-heptadecenyl)resorcinol (8), (13E,17E,21E)-polypodo-13,17,21-trien-3,8-diol (9), (13E,17E, 21E)-8-hydroxypolypodo-13,17,21-trien-3-one (10), mangiferolic acid (11), and 5-(13'Z-nanodecenyl)resorcinol (12) (๑ Fig. 1), were isolated from commercially available $C$. wightii gum resin at NCNPR. The identity and purity of all of the standard compounds were confirmed by chromatographic (TLC, HPLC) methods and by analysis and comparison of the spectroscopic data (IR, 1D- and 2D-NMR, HR-ESI-MS) with the published data [3]. The following \% purities were calculated for compounds 1-12: 99.65\%, 98.29\%, 96.41\%, 85.01\%, 93.18\%, 92.13\%, 98.78\%, 98.86\%, 91.48\%, 95.71\%, $94.93 \%$, and $96.19 \%$, respectively. Acetonitrile, methanol, and acetic acid were of HPLC grade and purchased from Fisher Scientific.
Different gum resin samples of $C$. wightii, C. myrrha, A. nilotica, $M$. indica, and $A$. indica were collected from different authentic and commercial sources in Pakistan and India. Some of the commercial samples were also obtained from USA. All of the samples of $C$. wightii were gum resin agglomerates. Out of twenty-two samples, one sample was the ethyl acetate extract of the $C$. wightii gum resin (EtOAc extract). Different $C$. wightii samples and related plant samples were assigned the following code numbers: C. wightii (NCNPR \#4997), C. wightii (NCNPR \#4998), C. wightii (NCNPR \#4999), C. wightii (NCNPR \#7764), C. wightii (NCNPR \#4985), M. indica (NCNPR \#5000), M. indica (NCNPR \#7761), M. indica (NCNPR \#7762), $M$. indica (NCNPR \#6517), M. azadirachta (NCNPR \#7763), and A. nilotica (NCNPR \#7760). All were obtained from India. C. wightii (NCNPR \#2567), C. wightii (NCNPR \#5772), C. wightii (NCNPR \#5774), C. wightii (NCNPR \#5775), C. wightii (NCNPR \#5776), C. wightii (NCNPR \#5777), C. wightii (NCNPR \#5778), C. wightii (NCNPR \#5780), C. wightii (NCNPR \#5781), C. wightii (NCNPR \#5782), C. wightii (NCNPR \#5783), C. wightii (NCNPR \#5784), C. wightii (NCNPR \#5785), C. wightii (NCNPR \#5786), C. wightii (EtOAc extract), and A. nilotica (NCNPR \#5773). These were obtained from Pakistan. C. myrrha (NCNPR \#2092) was procured from William Ware House. C. myrrha (NCNPR \#3550) was purchased from Frontier Natural Products Corporation.

Voucher specimens of all of the samples were deposited at the National Center for Natural Product Research (NCNPR), University of Mississippi, Mississippi, USA.

\section{Sample preparation}

Homogenized gum resin sample $(100 \mathrm{mg}$ ) was accurately weighed and mixed with $2.5 \mathrm{~mL} \mathrm{MeOH}$, and sonicated for $20 \mathrm{~min}$. It was then centrifuged for $10 \mathrm{~min}$ at $3000 \mathrm{rpm}$. The supernatant was transferred into a $10.0-\mathrm{mL}$ volumetric flask. The procedure was repeated three times, and all extracts were combined. The final volume was adjusted with $\mathrm{MeOH}$ to $10.0 \mathrm{~mL}$. The extract was thoroughly mixed and filtered with a $0.45-\mu \mathrm{m}$ nylon syringe filter prior to HPLC analysis. The first filtrate, $1 \mathrm{~mL}$, was discarded and the remaining filtrate was collected in an LC vial for further analysis.

All of the powdered gum resin samples were extracted on the basis of this protocol except one $C$. wightii gum resin sample; this sample was previously extracted in ethyl acetate (EtOAc extract) and used in our previous phytochemical study [3]. The EtOAc extract of the gum resin was taken $(1 \mathrm{mg})$, diluted 10 times, and then subjected to the above procedure to make up the final volume in $10 \mathrm{~mL}$ of methanol solution.

\section{Preparation of standard solution}

Stock solutions of E- and Z-guggulsterones were prepared in methanol at a concentration of $1.0 \mathrm{mg} / \mathrm{mL}$. Ten different concentration levels were used to record the calibration curve. The range of the calibration curve was $0.5-250 \mu \mathrm{g} / \mathrm{mL}$ for HPLC-UV analysis. Stock solution of the individual standard was prepared at a concentration of $1.0 \mathrm{mg} / \mathrm{mL}$ in methanol. $50 \mu \mathrm{L}$ of each standard solution was mixed to prepare a standard mixture of all of the twelve standard compounds for chemical fingerprinting analysis.

\section{Validation procedure}

The newly developed HPLC method was validated in terms of specificity, precision, accuracy, and linearity according to ICH guidelines [12]. The assay method precision was measured by interday and intraday studies. Known amounts of the E- and Z-gug- 
gulsterones, each $500 \mu \mathrm{g}$, were spiked to evaluate the accuracy of the assay method. LOD and LOQ were determined by injecting a series of dilute solutions with known concentrations. LOD and LOQ were defined as the signal-to-noise ratio equal to 3 and 10, respectively.

\section{Acknowledgements \\ $\nabla$}

This research was co-funded by the Pak-USAID project (Linkages of Centers for Chemical Sciences, Grant No.1-5/ILS-US/HEC/ 2004), and the United States Food and Drug Administration (FDA) Specific Cooperative Research Agreement Number 5 U01FD002 071-08. The authors are grateful to Dr. Vaishali C. Joshi, a plant taxonomist, for identification of the plant samples.

\section{Conflict of Interest}

\section{$\nabla$}

There is no conflict of interest to disclose.

\section{Affiliations}

${ }^{1}$ National Center for Natural Products Research, University of Mississippi, University, MS, USA

2 Division of Pharmacognosy, Department of BioMolecular Sciences, School of Pharmacy, University of Mississippi, University, MS, USA

${ }^{3}$ H. E.J. Research Institute of Chemistry, International Center for Chemical and Biological Sciences, University of Karachi, Karachi, Pakistan

${ }^{4}$ Department of Chemistry, College of Science, King Saud University, Riyadh, Saudi Arabia

${ }^{5}$ Department of Basic Sciences, DHA Suffa University, Karachi, Pakistan

\section{References}

1 Soni V. IUCN final project report of in-situ conservation of Commiphora wightii a red-listed medicinal plant species of Rajasthan State, India. 2008: 1-39. Available at: https://cmsdata.iucn.org/downloads/final_ project_report_dr_vineet_soni.pdf. Accessed November 30, 2015.

2 Ichikawa H, Aggarwal BB. Guggulsterone inhibits osteoclastogenesis induced by receptor activator of nuclear factor-kappaB ligand and by tumor cells by suppressing nuclear factor-kappaB activation. Clin Cancer Res 2006; 12: 662-668

3 Ahmed R, Ali Z, Wu Y, Kulkarni S, Avery MA, Choudhary MI, Khan IA. Chemical characterization of a commercial Commiphora wightii resin sample and chemical profiling to assess for authenticity. Planta Med 2011; 77: 945-950

4 Zhu N, Rafi MM, DiPaola RS, Xin J, Chin CK, Badmaev V, Ghai G, Rosen RT, Ho CT. Bioactive constituents from gum guggul (Commiphora wightii). Phytochemistry 2001; 56: 723-727

5 Meselhy MR. Inhibition of LPS-induced NO production by the oleogum resin of Commiphora wightii and its constituents. Phytochemistry 2003; 62: 213-218

6 Francis JA, Raja SN, Nair MG. Bioactive terpenoids and guggulusteroids from Commiphora mukul gum resin of potential anti-inflammatory interest. Chem Biodivers 2004; 1: 1842-1853

7 Mesrob B, Nesbitt C, Misra R, Pandey RC. High-performance liquid chromatographic method for fingerprinting and quantitative determination of E- and Z-guggulsterones in Commiphora mukul resin and its products. J Chromatogr B Biomed Sci Appl 1998; 720: 189-196

8 Nagarajan M, Waszkuc TW, Sun J. Simultaneous determination of Eand Z-guggulsterones in dietary supplements containing Commiphora mukul extract (guggulipid) by liquid chromatography. J AOAC Int 2001; 84: $24-28$

9 Haque I, Kumar M, Mukhopadhyay K. A rapid and simple UPLC-MS-MS based simultaneous determination of the medicinally important Eand Z-guggulsterone from oleogum-resins of naturally occurring Commiphora wightii plants. Chromatographia 2009; 70: 1613-1619

10 Hung T, Stuppner H, Ellmerer-Mueller EP, Scholz D, Eigner D, Manandhar MP. Steroids and terpenoids from the gum resin of Ailanthus grandis. Phytochemistry 1995; 39: 1403-1409

11 Hung T, Sturm S, Stuppner H. TLC and HPLC/MS analysis of the gum resin of Ailanthus grandis and related species. Sci Pharm 1996; 64: 471478

12 European Medicines Agency. ICH Topic Q2 (R1) Validation of Analytical Procedures: Text and Methodology. London: EMEA; 1995 